\title{
Ganglioside as a Therapy Target in Various Types of Cancer
}

\author{
Elmira Safaie Qamsari ${ }^{1}$, Alireza Nourazarian ${ }^{2,3 *}$, Salman Bagheri ${ }^{1}$, Morteza \\ Motallebnezhad $^{1}$
}

\begin{abstract}
Since their discovery in 1940, it has been well established that gangliosides are associated with a number of biological pathways and cellular processes such as growth, differentiation and toxin uptake. Gangliosides are glycosphingolipids containing neuraminic acid which are expressed on the plasma membrane of cells particularly in the nervous system. Heterogeneity and structural variation in the carbohydrate chains of gangliosides contributes to unique features of each of these molecules. Thirty five years ago it was discovered that aberrant glycosylation occurs in a variety of human cancers, including aberrant glycosylation of gangliosides. Ganglioside expression in terms of quality and quantity varies in different cancers and different roles may be played. Gangliosides, by affecting the immune system, including esxpression of cytokines and adhesion molecules, may inhibit anti-tumor mechanisms, as well as having direct impact on angiogenesis, cell movement and metastasis. It should be noted that different kinds of gangliosides do not all act by the same mechanisms.
\end{abstract}

Keywords: Gangliosides - cancer - immune system - biological pathways

Asian Pac J Cancer Prev, 17 (4), 1643-1647

\section{Introduction}

A class of diseases in which abnormal cells grow and multiply without control and have the ability to spread to the other parts of the body, are called cancer. Various factors and compounds are involved in creation, development and invasion of tumor. Gangliosides are one of compounds in natural cells of all animals. Gangliosides are glycosphingolipids containing neuraminic acid that exist naturally in the plasma membrane of cells but are differentially expressed in terms of quantity and quality in normal and tumor cells. Change in Gangliosides biosynthesis during malignant causes changes in the amount and type of Gangliosides expressed in many tumors (Cui et al., 2014). Changes in Gangliosides play an important role in the development and progression of abnormal reaction in the body (Fernandez et al., 2010) so that by various functions alters the biological effects of several treatment factors, and also affect mitogenic signaling cascade, growth factor and cell proliferation (Birkle et al., 2010).

Gangliosides are responsible for body's physiological actions such as embryogenesis and in the pathological conditions are effective in advance of tumor, its invasion and metastasis (Kwak et al., 2011).Variations in the carbohydrate structure and constructive ceramide of Gangliosides cause these compounds have different levels: for example, if the ganglioside is linked to compact neutral oligosacharid with its terminal sialic acid, it has more activity in weakening the immune system (Krengel and Bousquet, 2014). It is believed that Neu3, sialidase dependent to human plasma membrane, involved in the hydrolysis of Gangliosides contributes to cell differentiation and signal transduction through this hydrolyzation, playing a vital role in cell surface regulation. Profile Change of Gangliosides in cultured cells has shown its role in regulating cell proliferation and differentiation (Fukumoto et al., 2000). Various types of tumors express glycosphingo lipid antigens such as gangliosides that are present in many tumors and detectable with the monoclonal antibody (Doronin et al., 2014).

Gangliosides show different effects on tumor as following: Effect on metastasis and angiogenesis of tumor, regulation of cell motility and signaling, immune suppression (Zeng et al., 2000; Doronin et al., 2014)

\section{Gangliosides and Immune Suppression}

One of important roles of Ganglioside in the body is its effects on the immune system. Tumor cells protect themselves against immune cells using immunosuppressive factors, for example tumor surrounding inhibitors inhibit infiltrating function of lymphocytes (Elgert et al.,1998; Doronin et al., 2014). one of classes of immunosuppressant molecules that may play a role in tumor progression are 
Gangliosides (Doronin et al., 2014).

It has been observed that tumor cells throw out their Lysates, such as Gangliosides (Sialo sphingolipid). This phenomenon is called Shedding, Gangliosides antigens on the cell surface or in solution (in the cell's surrounding) act as a immunosuppressors and it is proposed that they protect tumor against the host immune system and also increase and enhance metastasis and angiogenesis (Dyatlovitskaya and Kandyba, 2006).

Inside body, Gangliosides conjugate with poorly tumorigenic cell and raise tumorigenicity (Caldwel et al., 2003). Tumors as Neuroblastoma, medulloblastoma and renal cell carcinoma, release their membrane Gangliosides into the surrounding of tumor, Gangliosides connect to target cells efficiently and effectively and inhibit immune system (Krengel and Bousquet, 2014). In fact, the Gangliosides that is shed into cell's surrounding from tumor cell exist in the form of micelles, monomers and membrane vesicles ( $\mathrm{Li}$ et al., 2013). More importantly, Gangliosides exhibits the ability to connect and interact with a variety of proteins, including signaling molecules present in the tumor environment. In addition, they are able to connect to other cell membranes leading to messaging or communicating with healthy cells (Doronin et al., 2014; Boligan et al., 2015).

The inhibitory effects of Gangliosides derived from tumor on cells of immune system in vivo have been appeared well. For example Gangliosides isolated from the plasma of cancer patients have inhibitory activity on the immune system and regulate various immune activities within the body (Shurin et al., 2001). Gangliosides have important roles through specific interaction of carbohydrates with proteins. For example, as receptor in cell-cell recognition and also by signaling regulatory proteins such as EGFR and VEGFR, they have function in membrane through interaction lateral (Krengel and Bousquet, 2014). Number of tumors, including neuroblastoma, melanoma, retinoblastoma and hepatoma has shown over expression of Gangliosides and release of Gangliosides into blood. For example, increased levels of GD2 and GM3 were found in serum analyses of patients with Neuroblastoma, retinoblastoma and hepatoma. Several reports suggest that the gangliosides like GD1a, GD1b, GD3 and GM3 help tumor for escaping from the immune system (Battula et al., 2012; Doronin et al., 2014; Krengel and Bousquet, 2014). The role of Tumor Gangliosides shown in Figure 1.

\section{Types of Gangliosides Effects On Cells of the Immune System}

Gangliosides antigens and their catabolites, through reaction with tyrosine kinase dependent to growth factor receptors or other kinase protein can alter cell signaling (Birkle et al., 2003).

GM2 gangliosides are involved in inhibiting the activity of several immune cells such as Th, NK (Biswas et al., 2006) and antigen and mitogen-stimulated $\mathrm{T}$ and $\mathrm{B}$ cell as well as inducers of apoptosis in $\mathrm{T}$ and $\mathrm{NK}$ cells (Zamfir et al., 2011). These compounds inhibit cytotoxicity effect of DC, Tcell and NK and prevent

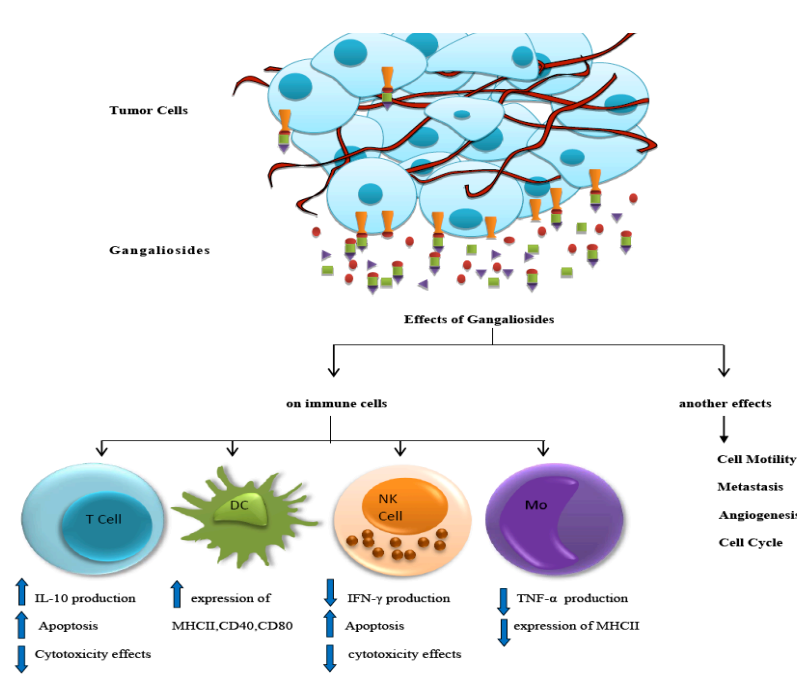

Figure 1. The different roles of Gangliosides in cancer cells

production of dendritic cells with activated function (Birkle et al., 2003).Ganglioside has inhibitory effect on monocytes. Gangliosides inhibits production of TNF- $\alpha$ from monocytes and also inhibit Antigen presentation by human monocytes (Krengel and Bousquet, 2014; Wang et al., 2015).

Gangliosides inhibit NK cells cytotoxic activity through interaction with SIGLEC-7 (sialicacid- binding immunoglobulin-like-lectin), Siglec-7 is as an inhibitory receptor on NK cells, which can connect to GD2 and GD3 and thus inhibit NK cells (Biswas et al., 2006).In addition to inhibiting NK cells cytotoxic activity, gangliosides inhibit IFN- $\gamma$ production by these cells (Battula et al., 2012). some of the Gangliosides that are present in bone marrow cause inhibition and change in hematopoiesis (Bharti and Singh, 2001). For example, patients with neuroblastoma that suffer bone marrow metastasis have often impaired hematopoiesis and pancitopenia is created in these patients (Niethammer and Handgretinger, 1995).

Immune suppression by Gangliosides may occur by several mechanisms, one of these mechanisms includes direct connection to the cytokine. One of the cytokines is IL-2 by connecting to which Gangliosides prevents interaction of IL-2 with IL-2R and inhibits proliferation of T cell Birkle et al., 2003).

In dealing with $\mathrm{T}$ cells, gangliosides have multiple effects, so as to prevent the activity and inhibit $\mathrm{T}$ cell antigen and mitogen-stimulated Bcells (Dyatlovitskaya and Kandyba,2006).T cell dysfunction is enhanced by Gangliosides GM2. While an antibody targets GM2, it can inhibit 60-50 percent of T cells apoptosis (Biswas et al., 2006). Gangliosides have shown the ability to inhibit NF-KB activation in cancerous cell of T-like renal cancer (Steiner et al., 2001). Furthermore, gangliosides can be affective in cytokine secretion from $\mathrm{T}$ cells, leading to increased production of IL10 from these cells (Caldwel et al., 2003).

It has recently been shown that glioblastoma cells, through distinct mechanisms that include interaction of gangliosides with CD70 and CD27, induce cell death in T cells .GD1a has inhibitory effect on APC dependent T 
cell proliferation. Gangliosides have inhibitory effects on NF-KB nuclear localization in dendritic cells. It has been noted that not only GD2 and GD3 lead to changes in integrin function, but also GD2 is highly effective in increased signals in the cells (Fukumoto et al., 2000; Caldwel et al., 2003). GD1a prevents expression of stimulatory molecules such as CD80 and CD40 on APC surface and decreases production of cytokines such as IL6, IL12 and TNFa (Büll et al., 2014).

\section{Ganglioside Effects on Cell Movement}

Gangliosides can affect movement and adhesion of cells. In studies the Neu3 is examined, it has been noted that Neu3 by reducing the inhibitory effect of GM3 / CD9 on the movement of cell, causes malignancy to increase and it has also been reported that addition of Gangliosides mixture to culture medium increases motitity in tumor cells (Zeng et al., 2000). It is noteworthy that GD3 increases tumor cell growth .It has been proved that Ganglioside increase movement of MCF7 cells by formation of Gb5monosialo complex with CD9 (Steelant et al., 2002). In a study the role of GM3 on c-Met messaging and cell movement and migration stimulation were studied; and 3 types of mouse liver cancer cell line were used in it. In that study, p4 was used for exclusive inhibition of GM3 and it was found that phosphorylation of c-Met and activity of signal transduction pathway AKT-PI3K was inhibited, while the increase in exogenous content of GM3 resulted in the increased GM3, phosphorylation of c-Met and pathway AKT-PI3K (Li et al., 2013).

It was demonstrated that the AKT-PI3K signal transduction pathway increases tumor genesis through Survival capacity in tumor cells. In another study that the mechanism of GM3 Silencing was examined, it was found that this mechanism clearly inhibits PTEN expression and subsequently activates AKT-PI3K in 4T1 cells. These results indicate that the migration and invasion of breast cancer cells is dependent GM3S, the study also stated that messaging GM3 inhibits expression of NF-AT1. It is proposed that prevention of NF-AT1 expression by activating the AKT-PI3K signal transduction pathway has important role in inhibition of migration and invasion, which is induced by GM3S Knockdown, in Maureen cells of breast (Gu et al., 2008). GM3, by forming a complex with $\alpha 3$ / CD9, inhibits the EGF receptor movement, also it has inhibitory effect on tyrosine kinase receptor, so reduction of GM3 rate prevents apoptosis, but raises the movement and proliferation (Hakomori, 2002).

\section{Ganglioside Roles in Metastasis}

Metastasis and angiogenesis are biochemically and clinically important in tumor progression (Wang et al., 2014).Tumor formation is as a result of spontaneous and uncontrolled proliferation of neoplastic cells, whereas tumor metastasis occurs when tumor cells release from primary tumor and continue reproduction in another region. Several factors affect this process; gangliosides as both donor molecules and stimulating molecules have different roles in the process of tumor progression and its
Gangliosides as Therapy Targets in Various Types of Cancer

metastasis. Gangliosides regulate adhesion and movement of the cells strongly, thus initiates tumor metastasis (Dyatlovitskaya and Kandyba, 2006; Nourazarian et al., 2014).

The process of metastasis starts with two main steps that include migration of tumor cells to extracellular environment and adhesion of them to other tissues; activity of Sphingolipid in both steps has been stated, noting that Sphingolipids are more involved in the second stage of metastasis. It has been also suggested that glycosylation and glycosphingolipid compounds and cialo sphingolipid (Gangliosides) are essential in the process of metastasis .Many studies have shown that tumor cells and metastatic cells are different in terms of gangliosides content. Different compositions of gangliosides present on cell membranes can affect the breadth and width of Spectrum, leading to changes in biology performance of the cell surface that is associated with the metastatic process (Labrada et al., 2010). Another role of ganglioside is to impact on tyrosine kinase growth factor receptor, For example GM3, by modulating tyrosine kinase growth factor receptor, regulates cell proliferation, invasion and metastasis of tumor cells ( $\mathrm{Li}$ et al., 2013). It has been shown that Metastatic melanoma cells express very high levels of GD3, compared to cells that express weak metastasic (Tringali et al., 2014). Accordingly, the role of GD3 in converting melanocytes to melanoma and strengthening metastatic is proposed (Gu et al., 2008).

\section{Ganglioside Roles in Angiogenesis}

As we know angiogenesis has important and critical role in tumor growth (Wang et al., 2013). Gangliosides have a major role in angiogenesis and it is proposed that they regulate the growth of the tumors by angiogenesis (Gately, 2000). These compounds show different roles in angiogenesis process, so that some have inhibitory effect of some are stimulus. Gangliosides shedding and releasing from tumor cell surface enhance angiogenesis (Labrada et al., 2010).

Although Gangliosides cannot induce angiogenesis but they have a synergistic effect with VEGF and fibroblast growth factor (Gately, 2000). prostaglandin E1 and copper ions Tumor cells release gangliosides from their surface along with membrane Vesicles, so serum of cancer patients has great amount of gangliosides; these Gangliosides affect tumor angiogenesis and targeting VEGF expression tumor cells as well as their levels so they release substantial amounts of gangliosides in the serum of cancer patients. The gangliosides affect tumor angiogenesis and metastasis by regulating expression of VEGF (Hakomori,2002). As reported, adding GD3 to culture medium stimulates the release of VEGF from glioma cells (Birkle et al., 2003). It has been proved that gangliosides like GD3 and GM2 enhance tumor-dependent angiogenesis (Horwacik and Rokita, 2015).

GM3 in EPEN (ependymoblastoma tumor cell line) inhibits tumor growth and angiogenesis, while GM2, GM1 and GD1synthesis cause high vascularization and increased production of VEGF through B4GalNAC-T transmission (Kwak et al., 2011). Ratio of gangliosides 
GM3 and GD3 affect angiogenesis process since GM3 inhibits angiogenesis and other gangliosides complexes containing (GD3, GM1, GM2, GT1b and GD1a) activates it (Krengel and Bousquet, 2014).

Gangliosides have important roles through specific interactions of carbohydrates with proteins. For example, they have function in cell-cell recognition as receptor and also in membrane by signaling regulatory proteins such as EGFR and VEGFR through intraction lateral. In Laboratory, Gangliosides enhances massaging of tyrosine kinase receptors and activates vascular endothelial cells (Liu et al., 2014).

\section{Gangliosides Function in Colon Cancer}

Colorectal cancer is the third most common cause of cancer deaths in the world. Epidemiological characteristics of colorectal cancer like incidence and distribution of age in different parts of the world is very extensive (Liu et al., 2014). Approximately five percent of the world's population suffers from colorectal cancer and every year 150,000 new cases of diagnosed cases is reported in United States of America. Industrial societies show a much higher risk of infection, while in developing societies this rate is so less. Unfortunately, incidence of this disease is increasing. Colon and rectum cancer progression is influenced by factors such as genetics, diet and environmental factors (Mousavi et al., 2009; Mehrabani et al., 2014). Aberrant glycosylation is a common feature of cancer cells (Reis et al., 2010). Aberrant glycosylation also occurs in colorectal cancer.

In a study glycosylation in tumor tissue was investigated in 13 patients with colorectal cancer and their results showed that glycosylation of glycosphingolipid changed during colorectal cancer progression, focalization levels in these patients increased, but acetylation and sulfatation reduced (Holst et al., 2013).

In other studies, it have been shown that levels of mRNA for sialidase in colon cancer increased (Kakugawa et al., 2002). As we know Sialidase have role in exclusive hydrolysis of Gangliosides and thus may play a role in cell differentiation and signaling. Evidence suggests that Sialidase overexpression in cancer cells is used to protect against programmed cell death and possible changes in Gangliosides. An overall increase in sialylation, often at the cell surface glycoprotein in malignant cells is observed (Miyagi et al., 2015).

Human Neu3 is a human ganglioside- specific sialidase with specific function and particular location and according to studies conducted, Sialidase gene transfer inhibits apoptosis in colon cancer cells and along with this BCL2 increased and caspase reduced (Kakugawa et al., 2002), NEU3 is markedly upregulated in colon cancer and it causing disorder in transmembrane signaling but decreasing reported in amount of NEU1 and NEU4 in colon cancer also NEU3 identified in apoptosis resistance mechanisms in colon cancer (Hata et al., 2015), also lactosil ceramide accumulation that is product of Sialidase is seen in colorectal cancer cells and decreases apoptotic cells by adding Lac-cer to culture medium of cells it during treatmnet with sodiumbutyrat. it was also reported that overexpression of Sialidase in colorectal cancer cells cause protection against programmed cell death or alters the Gangliosides content (Kakugawa et al., 2002). In addition to what mentioned, GD3 is also hydrolyzed very fast with increasing Neu3 and directs tumor cells to the inhibition of apoptosis expressed in colon cancer Kakugawa et al., 2002). GD3 as a bioactive lipid that targeting mitochondria and participate in cell death pathways and apoptosis. about cell death, many studies reported a novel role for GD3 in autophagy and in the regulation of lipid rafts microdomains (Garcia et al., 2015).

\section{Conclusion}

It can be concluded that gangliosides can be used as therapy targets for various types of cancer and it is recommended that further studies be conducted in this field in future.

\section{References}

Battula VL, Shi Y, Evans KW, et al (2012). Ganglioside GD2 identifies breast cancer stem cells and promotes tumorigenesis. J Clin Invest, 122, 2066-78.

Bharti AC, Singh SM (2001). Gangliosides derived from a T cell lymphoma inhibit bone marrow cell proliferation and differentiation. Int. Immunopharmacol, 1, 155-165.

Birkle S, Zeng G, Gao L, Yu RK, Aubry J (2003). Role of tumorassociated gangliosides in cancer progression. Biochimie, $\mathbf{8 5}, 455-63$.

Biswas K, Richmond A, Rayman P, et al (2006). GM2 expression in renal cell carcinoma: potential role in tumor-induced T-cell dysfunction. Cancer Res, 66, 6816-25.

Boligan KF, Mesa C, Fernandez LE, von Gunten S (2015). Cancer intelligence acquired (CIA): tumor glycosylation and sialylation codes dismantling antitumor defense. Cell Mol Life Sci, 72, 1231-48.

Büll C, den Brok MH, Adema GJ (2014). Sweet escape: sialic acids in tumor immune evasion. Biochim Biophys Acta, 1846, 238-46.

Caldwel S, Heitger A, Shen W, et al (2003) Mechanisms of ganglioside inhibition of APC function. J Immunol, 171, 1676-83.

Cui XB, Peng H, Li S, et al (2014). Prognostic value of PLCE1 expression in upper gastrointestinal cancer: a systematic review and meta-analysis. Asian Pac J Cancer Prev, 15, 9661-6.

Doronin II, Vishnyakova PA, Ponomarev ED, et al (2014). Ganglioside GD2 in reception and transduction of cell death signal in tumor cells. BMC Cancer, 14, 295.

Dyatlovitskaya EV, Kandyba AG (2006). Sphingolipids in tumor metastases and angiogenesis. Biochemistry (Moscow), 71, 347-53.

Elgert KD, Alleva DG, Mullins DW (1998). Tumor-induced immune dysfunction: the macrophage connection.J Leukoc Biol, 64, 275-90.

Fernandez LE, Gabri MR, Guthmann MD, et al (2010). NGcGM3 ganglioside: a privileged target for cancer vaccines. Clin Dev Immunol, $\mathbf{8}, 1-8$.

Fukumoto S, Mutoh T, Hasegawa T (2000). GD3 synthase gene expression in $\mathrm{PC} 12$ cells results in the continuous activation of TrkA and ERK1/2 and enhanced proliferation. $J$ Biol Chem. 275, 5832-8.

Garcia-Ruiz C, Morales A, Fernandez-Checa JC (2015). Glycosphingolipids and cell death: one aim, many ways. 
Apoptosis, 20, 607-20.

Gately S (2000). The contributions of cyclooxygenase- 2 to tumor angiogenesis. Cancer Metastasis Rev, 19, 19-27.

Gu Y, Zhang J, Mi W (2008). Silencing of GM3 synthase suppresses lung metastasis of murine breast cancer cells. Breast Cancer Res, 10, 1.

Hakomori S (2002). Glycosylation defining cancer malignancy: new wine in an old bottle. Proc Natl Acad Sci U S A. 99 , 10231-3.

Hata K, Tochigi T, Sato I, et al (2015). Increased sialidase activity in serum of cancer patients: Identification of sialidase and inhibitor activities in human serum. Cancer Sci, 106,383-9.

Holst S, Stavenhagen K, Balog CI, et al (2013) . Investigations on aberrant glycosylation of glycosphingolipids in colorectal cancer tissues using liquid chromatography and matrixassisted laser desorption time-of-flight mass spectrometry (MALDI-TOF-MS). Mol Cell Proteomics, 12, 3081-93.

Horwacik I, Rokita H (2015). Targeting of tumor-associated gangliosides with antibodies affects signaling pathways and leads to cell death including apoptosis. Apoptosis, 20, 679-88.

Kakugawa Y, Wada T, Yamaguchi K, et al (2002). Up-regulation of plasma membrane-associated ganglioside sialidase (Neu3) in human colon cancer and its involvement in apoptosis suppression. Natl Acad Sci U S A, 99, 10718-23.

Krengel U, Bousquet PA (2014). Molecular recognition of gangliosides and their potential for cancer immunotherapies. Front Immunol, 21, 325.

Kwak DH, Seo BB, Chang KT, Choo YK (2011). Roles of gangliosides in mouse embryogenesis and embryonic stem cell differentiation. Exp Mol Med, 43, 379-388.

Labrada M, Clavell M, Bebelagua Y (2010). Direct validation of NGcGM3 ganglioside as a new target for cancer immunotherapy. Expert Opin Biol Ther, 10, 153-62.

Li Y, Huang X, Zhong W, Zhang J, Ma K (2013). Ganglioside GM3 promotes HGF-stimulatedmotility of murine hepatoma cell through enhanced phosphorylation of cMet at specific tyrosine sites and PI3K/Akt-mediated migration signaling, Mol Cell Biochem, 382, 83-92.

Liu Y, Wondimu A, Yan S, Bobb D, Ladisch S (2014). Tumor gangliosides accelerate murine tumor angiogenesis. Angiogenesis, 17, 563-71.

Mehrabani D, Shamsdin SA, Dehghan A, Safarpour A (2014). Clinical significance of serum vascular endothelial growth factor and complement $3 \mathrm{a}$ levels in patients with colorectal cancer in southern Iran. Asian Pac J Cancer Prev, 15, 9713-7.

Miyagi T, Takahashi K, Shiozaki K, Yamaguchi K, Hosono M (2015). Plasma membrane-associated sialidase confers cancer initiation, promotion and progression. Adv Exp Med Biol, 842, 139-45.

Mousavi SM,Ramazani R,Gouya MM, et al (2009). Cancer incidence and mortality in Iran. Ann Oncol. 20, 556-563.

Niethammer D, Handgretinger R (1995). Clinical strategies for the treatment of neuroblastoma. Eur J Cancer. 31, 568-71.

Nourazarian AR, Kangari P Salmaninejad A (2014). Roles of oxidative stress in the development and progression of breast cancer. Asian Pac J Cancer Prev, 15, 4745-51.

Reis CA, Osorio H, Silva L, Gomes C, David L (2010). Alterations in glycosylation as biomarkers for cancer detection. J Clin Pathol, 63, 322-9.

Shurin GV, Shurin MR, Bykovskaia S, et al (2001). Neuroblastoma-derived gangliosides inhibit dendritic cell generation and function. Cancer Res, 61, 363-9.

Steelant WF, Kawakami Y, Ito A, et al (2002). Monosialyl-Gb5 organized with cSrc and FAK in GEM of human breast carcinoma MCF-7 cells defines their invasive properties.
FEBS letters, 531, 93-8.

Steiner T, Junker U, Henzgen B, et al (2001). Interferon-alpha suppresses the antiapoptotic effect of NF-kB and sensitizes renal cell carcinoma cells in vitro to chemotherapeutic drugs. Eur Urol, 39, 478-83.

Tringali C, Silvestri I, Testa F, et al (2014). Molecular subtyping of metastatic melanoma based on cell ganglioside metabolism profiles. BMC Cancer, 14, 560.

Wang Y, Cui Y, Cao F, et al (2015). Ganglioside GD1a suppresses LPS-induced pro-inflammatory cytokines in RAW264.7 macrophages by reducing MAPKs and NF- $x \mathrm{~B}$ signaling pathways through TLR4. Int Immunopharmacol, 28, 136-45.

Wang Z, Ma L, Zhang XM, Zhou ZX (2014). Risk of lymph node metastases from early gastric cancer in relation to depth of invasion: experience in a single institution. Asian Pac J Cancer Prev, 15, 5371-5.

Zamfir AD, Serb A, Vukeli Z, et al (2011). Assessment of the molecular expression and structure of gangliosides in brain metastasis of lung adenocarcinoma by an advanced approach based on fully automated chip-nanoelectrospray mass spectrometry. J Am Soc Mass Spectrom, 22, 2145-59.

Zeng G, Gao L, Birkle S, Yu RK (2000). Suppression of ganglioside GD3 expression in a rat F-11 tumor cell line reduces tumor growth, angiogenesis, and vascular endothelial growth factor production, Cancer Res, 60 , $6670-7$. 